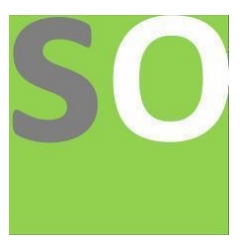

Article title: Visual Symmetrical effects on the human brain

Authors: Shubhangi Sharma[1]

Affiliations: Keshav mahavidyalaya, University of Delhi[1]

Orcid ids: 0000-0002-6284-073X[1]

Contact e-mail: shubhangi220601@gmail.com

License information: This work has been published open access under Creative Commons Attribution License $\mathrm{http}: / / c r e a t i v e c o m m o n s . o r g / l i c e n s e s / b y / 4.0 /$, which permits unrestricted use, distribution, and reproduction in any medium, provided the original work is properly cited. Conditions, terms of use and publishing policy can be found at https://www.scienceopen.com/.

Preprint statement: This article is a preprint and has not been peer-reviewed, under consideration and submitted to ScienceOpen Preprints for open peer review.

Links to data: https://docs.google.com/document/d/1MtUbkn1nfWR9y8-sIWD6plvzw0ZiytLURaVwRpR0wfw DOI: 10.14293/S2199-1006.1.SOR-.PPV7ZQW.v1

Preprint first posted online: 08 January 2022

Keywords: symmetry, human brain , asymmetry 


\title{
Visual Symmetrical effects on the human brain
}

\author{
Shubhangi Sharma
}

\begin{abstract}
$\underline{\text { Abstract }}$
The human brain is a very complicated machine. In this paper, we present a new way of interpretation that explains how the brain reacts to the presented symmetrical figures.

\section{Introduction}

An object has symmetry if there is an operation or transformation that maps the figure/object onto itself. Symmetry is also been the organisational structure of living organisms. It has been seen that the human brain often sees symmetrical objects in the environment easily. Should we conclude that the human brain doesn't want to process asymmetrical information or a kind of survival skill? Even a spider's brain relies upon symmetry for its survival. For example, a white crab spider lives in a symmetrical flower for its food. Is it a very complex machine? it has the best efficiency as compared to any other machine.

From the evolution of several thousand years, our brains have evolved to focus on symmetrical objects. Let it be anything like prey or predator. For instance, if a lion has a pretty symmetrical face, and our brain detects it coming, it would immediately activate our fight or flight response to continue further.

The importance of symmetry to the human mind is that it facilitates image processing and makes it easy to explore the object and then take further action. 


\section{Method}

To get started right away, I've made a google form. This form asks people to select one of the options from the given two images. One is symmetrical and the other is asymmetrical. Participants must select one of the images and submit the form.

\section{$\underline{\text { Expected result }}$}

It is been expected that most of the participants will choose symmetrical pictures instead of asymmetrical pictures.

\section{Google form}

\section{simple questions}

What you have to do is just select one image from the two options.

* Required

Email *

Your email

your name *

Your answer 
Which one will you pick up? *
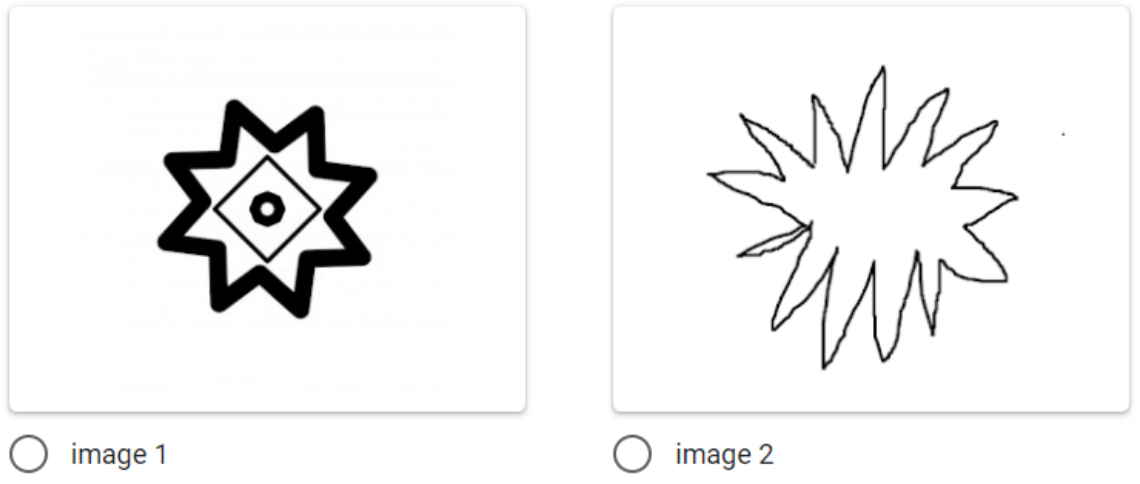

Which one will you pick up? *

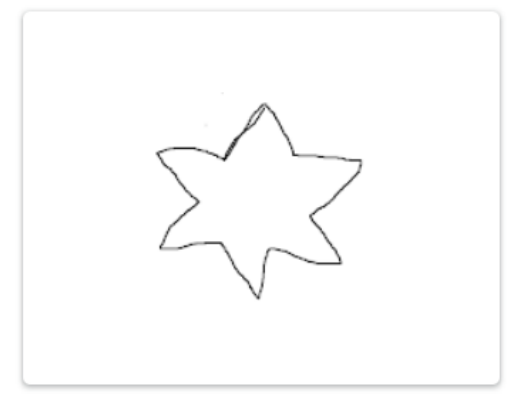

image 1

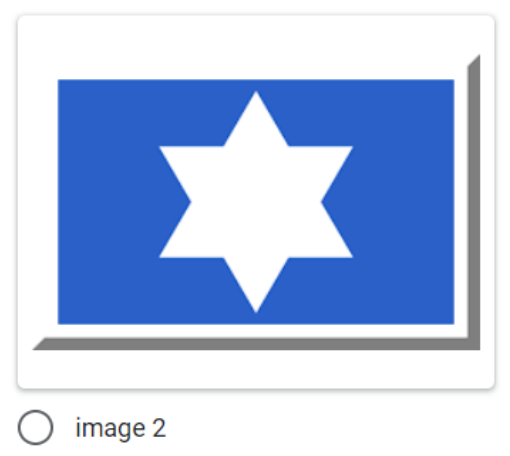

Which one will you pick up? *

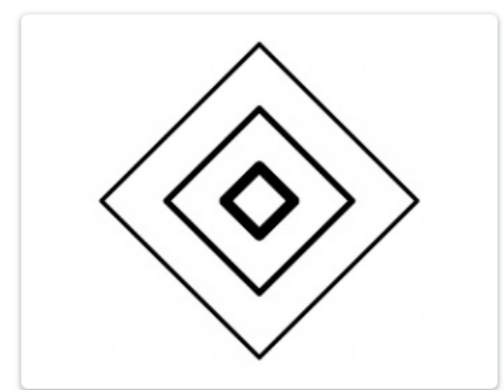

$\bigcirc$ image 1

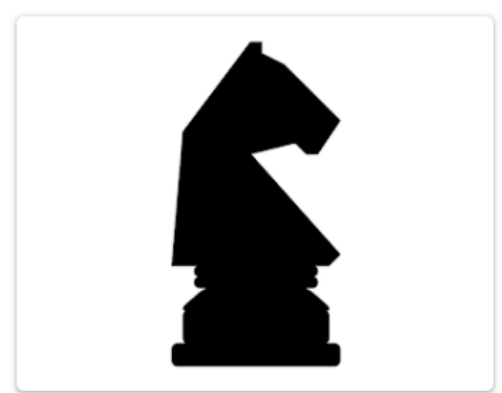

image 2 
Which one will you pick up? *
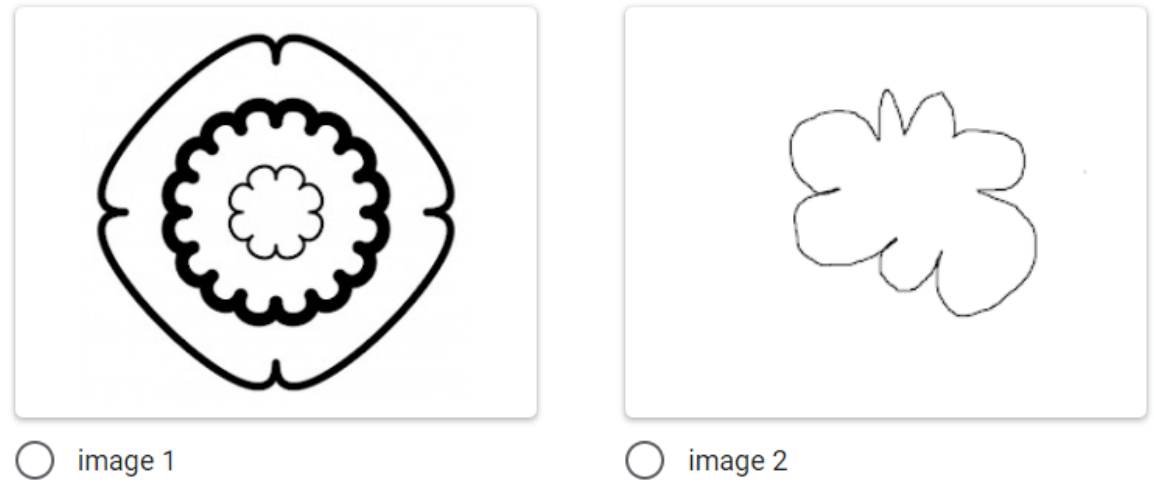

which one will you pick up *
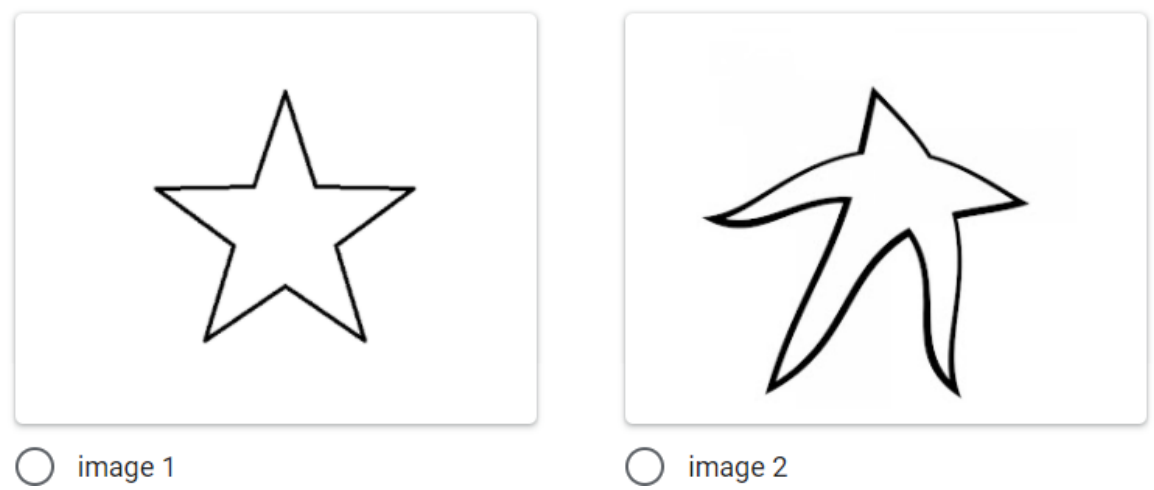
Which one will you pick up? *
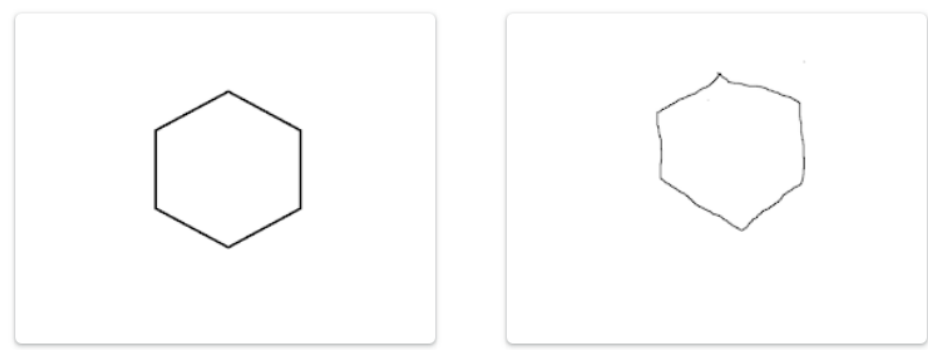

image 1

image 2

Send me a copy of my responses.

Submit

Never submit passwords through Google Forms.

3. reCAPTCHA
Privacy Terms

This form was created inside of Keshav Mahavidyalaya. Report Abuse

Google Forms 


\section{$\underline{\text { Observations }}$}

your name

50 responses

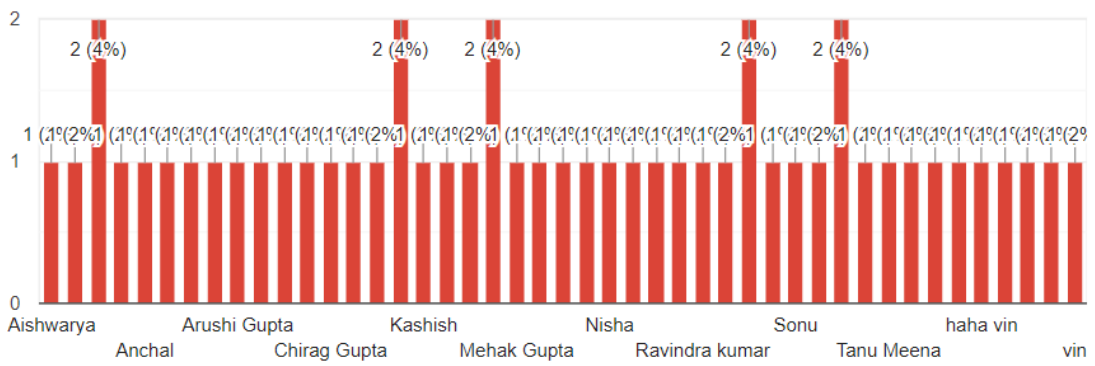

Which one will you pick up?

50 responses

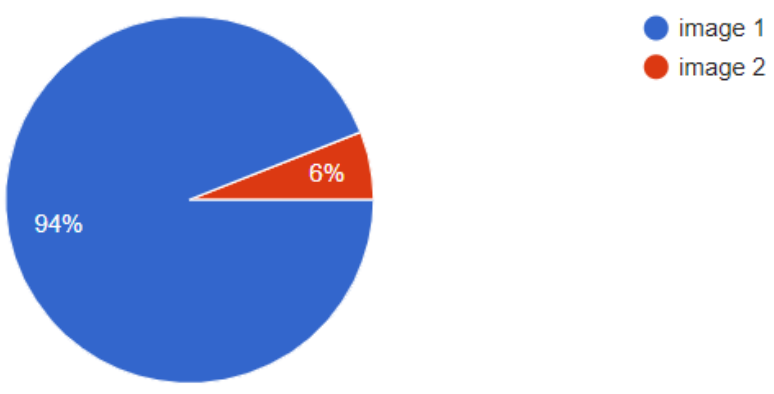


Which one will you pick up?

50 responses

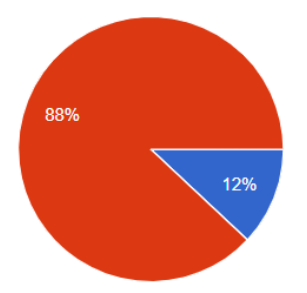

image 1

image 2

Which one will you pick up?

50 responses

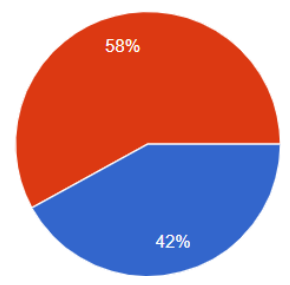

image 1

image 2

Which one will you pick up?

50 responses

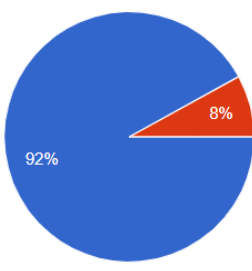

- image 1

image 2

which one will you pick up

50 responses

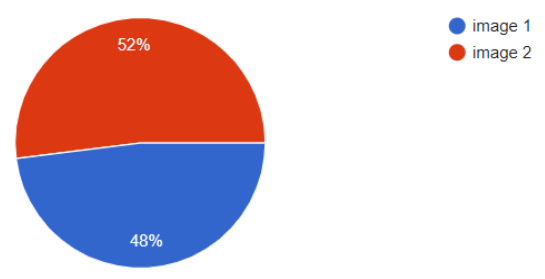


Which one will you pick up?

50 responses

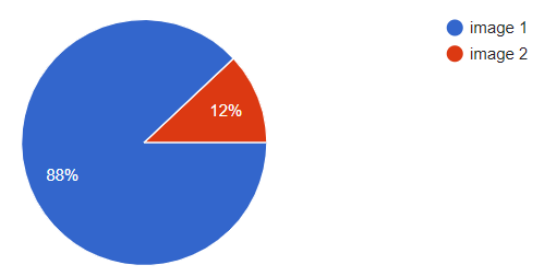

\section{$\underline{\text { Results }}$}

It has been verified that most of the people who participated in this experiment have chosen symmetrical images as expected. That means that our brains have improvised to such extents that we prefer to choose visual symmetries instead of asymmetries. That explains why we suddenly detect faces in every household object like drawers and almirahs, etc., to ensure our survival, every brain works very very hard.

\section{Conclusion}

The human brain prefers symmetry over asymmetry. The human brain doesn't prefer on giving attention to asymmetrical objects because of the following factors:

1. Evolutionary survival techniques.

2. It reduces the strain on the eye.

3. It makes the brain easier to understand the presented concept.

4. It reduces the effort to make the decision.

\section{$\underline{\text { References }}$}

1. https://www.mdpi.com/2073-8994/6/4/975 Brain Activity in Response to Visual Symmetry, Authors: Marco Bertamini and Alexis D J Makin, Department of Psychological Sciences, University of 
Liverpool, Eleanor Rathbone Building, Bedford Street South, Liverpool L69 7ZA, UK.Symmetry 2014, 6(4), 975-996; https://doi.org/10.3390/sym6040975

2. Symmetry and asymmetry analysis and its implications to computer-aided diagnosis: A review of the literature, by Sheena Lin Xu; Department of Biomedical Informatics, Columbia University, New York, NY 10032, USA;https://doi.org/10.1016/j.jbi.2009.07.003

3. Marais PC, et al. Visualising cerebral asymmetry. Lecture notes in computer science; 1996. 